\title{
Trajectory of Cardiac Catheterization for Acute Coronary Syndrome and Out-of-Hospital Cardiac Arrest During the COVID-19 Pandemic
}

\author{
Pooja S. Desai ${ }^{a}$, e, Elias J. Fanous ${ }^{a}$, Weiyi Tan ${ }^{b, c}$, James Lee ${ }^{d}$, Tri Trinh ${ }^{d}$, \\ Asim M. Rafique ${ }^{\mathrm{b}}$, Rushi V. Parikh ${ }^{\mathrm{b}}$, Marcella Calfon Press ${ }^{\mathrm{b}}$
}

\begin{abstract}
Background: We sought to investigate the trajectory of cardiac catheterizations for acute coronary syndrome (ACS) and out-of-hospital cardiac arrest (OHCA) during the pre-isolation (PI), strict-isolation (SI), and relaxed-isolation (RI) periods of the coronavirus disease 2019 (COVID-19) pandemic at three hospitals in Los Angeles, CA, USA.

Methods: A retrospective analysis was conducted on adult patients undergoing urgent or emergent cardiac catheterization for suspected ACS or OHCA between January 1, 2020 and June 2, 2020 at three hospitals in Los Angeles, CA, USA. We designated January 1, 2020 to March 17, 2020 as the PI COVID-19 period, March 18, 2020 to May 5, 2020 as the SI COVID-19 period, and May 6, 2020 to June 2, 2020 as the RI COVID-19 period.
\end{abstract}

Results: From PI to SI, there was a significant reduction in mean weekly cases of catheterizations for non-ST elevation myocardial infarction/unstable angina (NSTEMI/UA) (8.29 vs. 12.5, P =0.019), with all other clinical categories trending downwards. From SI to RI, mean weekly cases of catheterizations for total ACS increased by $17 \%$, NSTEMI/UA increased by $27 \%$, and OHCA increased by $32 \%$, demonstrating a "rebound effect".

Conclusions: Cardiac catheterizations for ACS and NSTEMI/UA exhibited a "rebound effect" once social isolation was relaxed.

Keywords: COVID-19; Acute coronary syndrome; Cardiac catheterization; Cardiac arrest

Manuscript submitted August 15, 2020, accepted August 29, 2020

Published online December 11, 2020

aDepartment of Internal Medicine, UCLA Medical Center, Los Angeles, CA, USA

${ }^{b}$ Division of Interventional Cardiology, UCLA Medical Center, Los Angeles, CA, USA

'Division of Congenital Cardiology, UCLA Medical Center, Los Angeles, CA, USA

dUCLA Cardiology Group, Henry Mayo Hospital, Valencia, CA, USA ${ }^{\mathrm{e} C}$ Corresponding Author: Pooja S. Desai, Department of Internal Medicine, UCLA Medical Center, 757 Westwood Avenue, Suite 7501, Los Angeles, CA, USA.Email: pdesai@mednet.ucla.edu

doi: https://doi.org/10.14740/cr1149

\section{Introduction}

Recent studies demonstrated a worrisome decline in cardiac catheterizations for acute coronary syndrome (ACS) during the initial peak of the coronavirus disease 2019 (COVID-19) pandemic [1-4]. Once the incidence of new COVID-19 cases appeared to decrease, isolation orders were relaxed across the country. We sought to investigate if this relaxed-isolation (RI) period was associated with an increase in cardiac catheterizations for ACS and out-of-hospital cardiac arrest (OHCA) requiring catheterization lab activation, thereby demonstrating a "rebound effect" following the first peak of COVID-19.

\section{Materials and Methods}

We performed a retrospective analysis of adult patients undergoing urgent or emergent cardiac catheterization for suspected ACS or OHCA between January 1, 2020 and June 2, 2020 at three hospitals in Los Angeles, CA, USA. We designated January 1, 2020 to March 17, 2020 as the pre-isolation (PI) COVID-19 period, March 18, 2020 to May 5, 2020 as the strict-isolation (SI) COVID-19 period, and May 6, 2020 to June 2, 2020 as the RI COVID-19 period. The Institutional Review Board of University of California, Los Angeles, approved this study and waived patient consent given the retrospective nature of the study. This study was conducted in compliance with all the applicable institutional ethical guidelines for the care, welfare and use of animals.

We included all adult (age $\geq 18$ years) patients with cardiac catheterizations for ACS, ST-elevation myocardial infarction (STEMI) activations, true STEMI, non-STEMI/unstable angina (NSTEMI/UA), and OHCA. STEMI activation was defined as a catheterization lab activation for a presumed STEMI or OHCA. ACS was defined as true STEMI or NSTEMI/UA. True STEMI was defined by the presence of a culprit lesion on angiogram. For all cases, we collected baseline demographic and cardiovascular risk factor data.

We compared cardiac catheterization volume for each clinical category (ACS, STEMI activation, true STEMI, NSTEMI/ UA, OHCA) for each time period. One-way analysis of variance (ANOVA) or Fisher's exact tests were used to evaluate differences between groups of variables, as appropriate. We 
Table 1. Demographics and Clinical Characteristics of Patients Undergoing Urgent or Emergent Cardiac Catheterization for Each Clinical Scenario in the Time Period Pre-Isolation (January 1, 2020 to March 17, 2020), Strict-Isolation (March 18, 2020 to May 5 , 2020), and Relaxed-Isolation (May 6, 2020 to June 2, 2020)

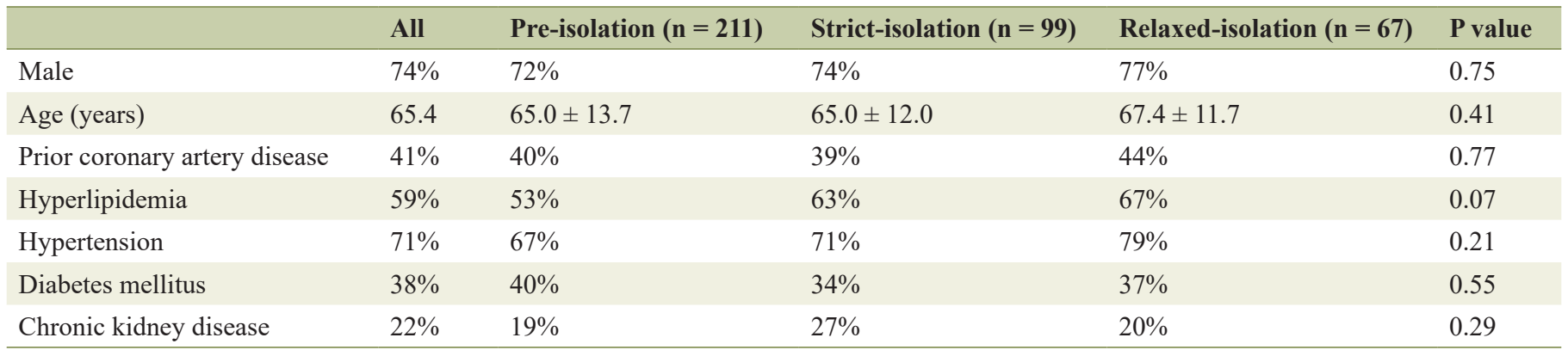

performed one-way ANOVA to evaluate if cases per week of ACS, STEMI activation, STEMI, NSTEMI/UA, and OHCA differed across PI, SI, and RI. Post hoc analysis was completed using Tukey's test. All statistical analyses were completed using STATA 16.1 MP (College Station, TX, USA), and a P value $<0.05$ was considered statistically significant.

\section{Results}

Overall, 377 patients were included in this study, with 211 patients during PI, 99 patients during SI, and 67 patients during RI. Baseline demographic and clinical characteristics were similar among the three groups (Table 1). There was a statistically significant difference in cardiac catheterization cases for NSTEMI/UA between groups $(\mathrm{P}=0.025$ for interaction (ANOVA)). This was driven by a significant difference in the mean number of cases per week of NSTEMI/UA in SI relative to PI (8.29 vs. $12.5, \mathrm{P}=0.019)$. There were no statistically significant differences in the other clinical scenarios (ACS, STEMI activation, OHCA) in SI relative to PI. However, trends toward an increase in cases in RI relative to SI were observed in ACS, NSTEMI/UA, and OHCA. Specifically, from SI to RI, the mean weekly number of catheterization cases for ACS increased by $17 \%$, NSTEMI/UA increased by $27 \%$, and OHCA increased by $32 \%$ (Fig. 1 ).

\section{Discussion}

To the best of our knowledge, there have been no studies investigating the impact of isolation relaxation on cardiac catheterizations for ACS and OHCA. As of June 2, 2020, Los Angeles had suffered 59,144 cases of COVID-19, comprising more than one-third of California's total cases [5]. The three hospitals included in this study serve a population that is diverse in socioeconomic status, race/ethnicity, and health literacy from West Los Angeles to the Santa Clarita Valley. Our primary objective was to investigate patterns in ACS and OHCA in this impacted population as isolation policies and perceptions relaxed. We found that each clinical category (ACS, STEMI activation, true STEMI, NSTEMI/UA, OHCA) initially decreased as isolation measures intensified. Our study's major findings were that ACS and NSTEMI/UA later increased as

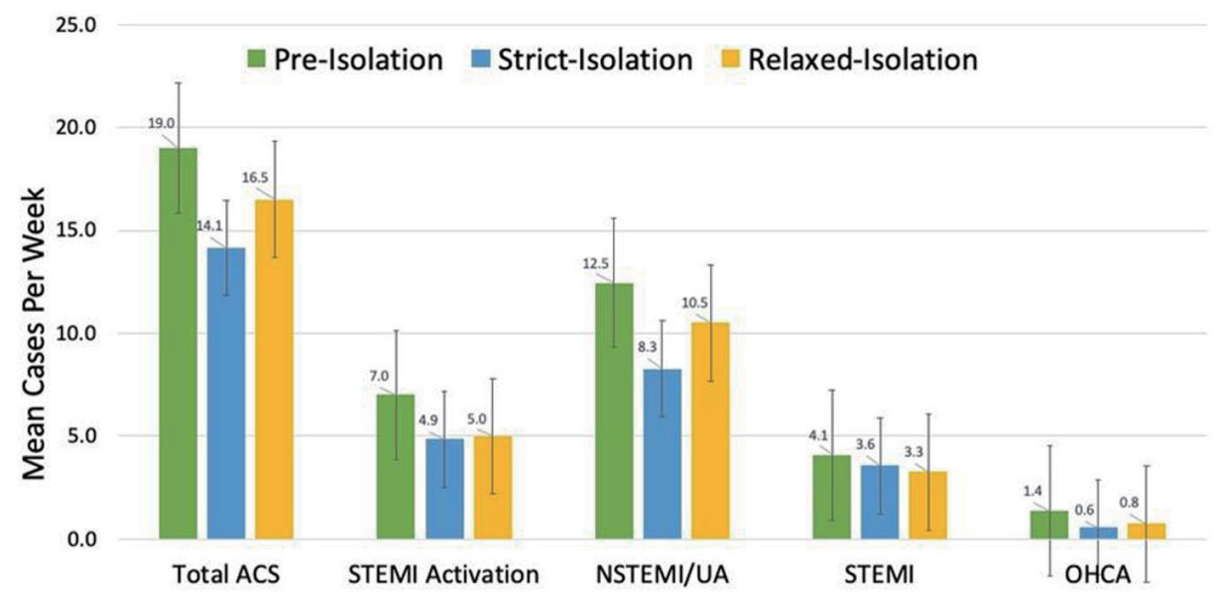

Figure 1. Mean number of cases per week for each clinical scenario during the time periods pre-isolation (January 1, 2020 to March 17, 2020), strict-isolation (March 18, 2020 to May 5, 2020), and relaxed-isolation (May 6, 2020 to June 2, 2020). Standard error bars are shown. *Statistically significant difference with a P value < 0.05. ACS: acute coronary syndrome; NSTEMI: non-ST elevation myocardial infarction/unstable angina; UA: unstable angina; OHCA: out-of-hospital cardiac arrest. 
isolation relaxed in May of 2020 in Los Angeles.

The rebound in total ACS is predominantly driven by the rise in cardiac catheterizations for NSTEMI/UA. Though these trends did not reach statistical significance, they are clinically meaningful in the context of prior studies that corroborate our findings of an initial decline in cardiac catheterizations for ACS and OHCA as isolation intensified [6, 7]. Given that our PI to SI findings are consistent with other published data, we believe the identified uptick in total ACS and NSTEMI/UA from SI to RI reflects an important clinical trend. We also found that OHCA increased from SI to RI, but because the total numbers of cases were low, meaningful conclusions cannot be drawn.

A number of reasons likely account for this "rebound effect" in NSTEMI/UA and ACS from SI to RI. First, major institutions developed public health campaigns encouraging patients to seek emergency care for chest pain and other symptoms [8]. Second, consistent with major societal guidelines [9-11], the three hospitals in this study initially developed protocols directing cardiologists to manage NSTEMI/UA cases medically in the absence of high-risk clinical features or hemodynamic instability during the initial months of COVID-19. The patients were managed medically with antiplatelet agents (aspirin $+\mathrm{P}_{2} \mathrm{Y}_{12}$ inhibitor) via an ischemia-guided strategy if they were deemed to be clinically stable and without refractory angina [12]. In May, these hospitals reverted to pre-COVID-19 protocols (i.e., routine cardiac catheterization for NSTEMI/ UA). Third, on May 5, Los Angeles Mayor Eric Garcetti announced a relaxation of the Shelter-At-Home orders in Los Angeles. Patients may have been reassured by these policy adjustments, and coupled with previously described public health education, felt more comfortable seeking medical attention. Finally, the rebound in NSTEMI/UA and ACS may be due to patients' coronary artery disease physiologically worsening during the months of isolation. Patients may have missed important appointments, failed to initiate important medications like antiplatelet agents and statins, and delayed revascularization for stable angina.

This study has several limitations, the primary one being its modest sample size. We suspect that the trends observed in our study would meet statistical significance in a larger, adequately powered study. This study is also limited in its geographic generalizability as it is focused on cardiac catheterizations at three hospitals in Los Angeles, CA. In other locations, there are wide-ranging differences in rates of COVID-19 infection, hospital-specific practice patterns, and public policies. Additionally, the present study is a qualitative, descriptive analysis designed to evaluate rates of cardiac catheterization for various clinical scenarios. Therefore, mortality rates or other secondary outcomes across time periods were not studied. Finally, this study did not investigate how reciprocal outof-hospital death from cardiac arrest changed during the same time periods, which would help clarify the hypothesis that patients delayed seeking care more than usual during SI.

In conclusion, cardiac catheterizations for ACS and NSTEMI/UA exhibited a "rebound effect" once social isolation was relaxed in Los Angeles. COVID-19 continues to wreak havoc on Los Angeles, which has already entered a second period of intensified isolation as cases rise precipitously
[13]. Further studies of how ACS and OHCA cases are affected by peaks and troughs of the ongoing pandemic are critical to formulating public policy, guiding medical management, and equipping patients with proper information for optimal safety.

\section{Acknowledgments}

None to declare.

\section{Financial Disclosure}

None to declare.

\section{Conflict of Interest}

None to declare.

\section{Informed Consent}

Not applicable.

\section{Author Contributions}

PSD contributed to data curation, formal analysis, investigation, methodology, software, and writing (original draft, review and editing); EJF contributed to conceptualization, data curation, and writing (original draft, writing-review and editing); WT contributed to conceptualization, data curation, formal analysis, investigation, methodology, software, validation, and supervision; JL and TT contributed to conceptualization, data curation, writing (review and editing); AMR contributed to conceptualization, formal analysis, investigation, methodology, project administration, and writing (review and editing); RVP contributed to conceptualization, project administration, writing (review and editing); and MCP contributed to conceptualization, investigation, and writing (original draft, review and editing).

\section{Data Availability}

The data supporting the findings of this study are available from the corresponding author upon reasonable request.

\section{References}

1. De Filippo O, D'Ascenzo F, Angelini F, Bocchino PP, Conrotto F, Saglietto A, Secco GG, et al. Reduced rate of hospital admissions for ACS during COVID-19 outbreak in Northern Italy. N Engl J Med. 2020;383(1):88-89.

2. Braiteh N, Rehman WU, Alom M, Skovira V, Breiteh N, Rehman I, Yarkoni A, et al. Decrease in acute coronary 
syndrome presentations during the COVID-19 pandemic in upstate New York. Am Heart J. 2020;226:147-151.

3. Tan W, Parikh RV, Chester R, Harrell J, Franco V, Aksoy $\mathrm{O}$, Dave R, et al. Single center trends in acute coronary syndrome volume and outcomes during the COVID-19 pandemic. Cardiol Res. 2020;11(4):256-259.

4. Bhatt AS, Moscone A, McElrath EE, Varshney AS, Claggett BL, Bhatt DL, Januzzi JL, et al. Fewer hospitalizations for acute cardiovascular conditions during the COVID-19 pandemic. J Am Coll Cardiol. 2020;76(3):280-288.

5. COVID-19 Public Health Los Angeles Data. http://dashboard.publichealth.lacounty.gov/covid19_surveillance_ dashboard/. Accessed July 14, 2020.

6. Garcia S, Albaghdadi MS, Meraj PM, Schmidt C, Garberich R, Jaffer FA, Dixon S, et al. Reduction in ST-Segment elevation cardiac catheterization laboratory activations in the United States during COVID-19 pandemic. J Am Coll Cardiol. 2020;75(22):2871-2872.

7. Rodriguez-Leor O, Cid-Alvarez B, Ojeda S, et al. Articulo original Impacto de la pandemia de COVID-19 sobre la actividad asistencial en cardiologia intervencionista en Espana Impact of the COVID-19 pandemic on interventional cardiology activity in Spain. REC Interv Cardiol. 2020;2(2):82-89.

8. The new pandemic threat: people may die because they're not calling 911 | American Heart Association. https:// newsroom.heart.org/news/the-new-pandemic-threat-people-may-die-because-theyre-not-calling-911. Accessed
July 14, 2020.

9. Mahmud E, Dauerman HL, Welt FG, Messenger JC, Rao SV, Grines C, Mattu A, et al. Management of acute myocardial infarction during the COVID-19 pandemic. J Am Coll Cardiol. 2020.

10. Wood DA, Sathananthan J, Gin K, Mansour S, Ly HQ, Quraishi AU, Lavoie A, et al. Precautions and procedures for coronary and structural cardiac interventions during the COVID-19 pandemic: guidance from Canadian Association of Interventional Cardiology. Can J Cardiol. 2020;36(5):780-783.

11. Welt FGP, Shah PB, Aronow HD, Bortnick AE, Henry TD, Sherwood MW, Young MN, et al. Catheterization laboratory considerations during the coronavirus (COVID-19) pandemic: from the ACC's Interventional Council and SCAI. J Am Coll Cardiol. 2020;75(18):2372-2375.

12. Amsterdam EA, Wenger NK, Brindis RG, Casey DE, Jr., Ganiats TG, Holmes DR, Jr., Jaffe AS, et al. 2014 AHA/ ACC guideline for the management of patients with nonST-elevation acute coronary syndromes: a report of the American College of Cardiology/American Heart Association Task Force on Practice Guidelines. Circulation. 2014;130(25):e344-426.

13. Reopening safer at work and in the community for control of COVID-19 moving the county of Los Angeles into stage 3 of California's pandemic resilience roadmap. https://www.gov.ca.gov/2020/04/03/at-newly-converted. Accessed July 19, 2020. 